\title{
Infantile tremor syndrome secondary to peroral vitamin B12 replacement therapy: a report of two cases with myoclonus
}

\author{
Beril Dilber ${ }^{1 \oplus}$, Gökçe Pınar Reis ${ }^{2 \oplus}$ \\ Departments of ${ }^{1}$ Pediatric Neurology and ${ }^{2}$ Pediatric Hematology-Oncology, Karadeniz Technical University Faculty of Medicine, \\ Trabzon, Turkey.
}

\begin{abstract}
Background. Abnormal movements such as tremors and myoclonus may be observed during both treatment and deficiency of vitamin B12, particularly in infants. Infantile tremor syndrome (ITS) is defined by the tetrad of pallor, developmental delay/regression, skin pigmentation, and brown scanty scalp hair.

Case. In this report, two cases with ITS aged less than one year who had myoclonic movements during vitamin peroral B12 treatment are discussed based on hematologic, neurological, and magnetic resonance images (MRI) findings, one of whom developed a whole-body tremor and rhythmic myoclonic movements, titubation, and restlessness in the hands and feet as well as diffuse cerebral atrophy on brain MRI.
\end{abstract}

Conclusion. The infants of mothers with nutritional vitamin B12 deficiency may develop sudden abnormal movements following peroral vitamin B12 therapy and that the differential diagnosis of these disorders is highly important for the prevention of long-term neurological sequela by treatment.

Key words: infantile tremor syndrome, myocolonic movement disorders, cerebral atrophy, peroral vitamin B12 therapy.

In Turkey, infantile vitamin B12 deficiency is mostly caused by a low intake of vitamin B12 in the diet. Vegetarian diet, pernicious anemia, and low socioeconomic level are common etiological factors associated with vitamin B12 deficiency in breastfeeding mothers and their children. ${ }^{1}$ Vitamin B12 begins accumulating in the infant within the first six months after birth while mothers often remain asymptomatic and the deficiency in the infant may not be noticed until the onset of neurological effects. ${ }^{2}$ Infantile symptoms including restlessness, nutritional problems, and neuromotor growth retardation become pronounced over time. Abnormal movements such as tremor, myoclonus, and choreoathetosis that are observed before or during the B12 vitamin injection treatment or

Beril Dilber

beriltem@gmail.com

Received 10th June 2020, revised 14th July 2020,

8th August 2020, 13th September 2020,

accepted 26th November 2020. due to cobalamin $(\mathrm{Cbl})$ deficiency are rare but have been previously reported, particularly in infants. ${ }^{3-8}$

In a recently published study, nutritional $\mathrm{Cbl}$ deficiency, particularly during infancy, was shown to cause a wide variety of abnormal movements such as involuntary eye deviations, eyelid twitching, and contraction in the whole or part of the body despite normal serum vitamin B12 levels and/or neurological development. ${ }^{9}$ In this report, we present two cases with Infantile tremor syndrome (ITS) aged less than one year old who developed myoclonic movements following peroral vitamin B12 replacement therapy.

\section{Case 1}

An eight-month-old girl with thin brown hair was admitted with hypotonia. Her parents were distant relatives and she had a low socioeconomic status. The patient was 
being breastfed and she had been born at term weighing $3.3 \mathrm{~kg}$ after an uncomplicated pregnancy and delivery. She started to smile at 3 months and had gained head control at 4 months of age (head circumference: $40 \mathrm{~cm}$ [25 p]). By the age of 6 months, she gradually became less active. On examination, she looked pale and hypotonic. Her head circumference was $41 \mathrm{~cm}(<3 p)$, body weight was $8 \mathrm{~kg}(10$ p), and body height was $68 \mathrm{~cm} \mathrm{(25p).} \mathrm{Cranial}$ nerve examination was normal. Although she was generally hypotonic, she had normal strength and brisk reflexes. A complete blood count $(\mathrm{CBC})$ indicated hemoglobin 8.9 (range, 12-17) g/dL, hematocrit $27.2 \%$ (range, $42-52 \%$ ), mean corpuscular volume (MCV) 85.1 (range, 80-94) fL, ferritin 100 (range, 23.9-336.2) $\mu \mathrm{g} / \mathrm{L}$, folic acid 10.56 (range, 3.1-19.9) $\mu \mathrm{g} / \mathrm{L}$, serum homocysteine 45 (range, 5-15) $\mu \mathrm{mol} / \mathrm{L}$, serum methylmalonic acid 4.85 (range, 0.0-3.60) $\mu \mathrm{mol} / \mathrm{L}$, and serum vitamin B12 46 (range, 126.5-505) ng/L. On the other hand, the mother's vitamin B12 level was also low (98 ng/L [range, 126.5-505] ng/L). Peripheral blood analysis indicated anisocytosis and hypersegmented neutrophils. Coronal T2-weighted images showed abnormal brain MRI findings including enlargement of frontotemporal subarachnoid spaces, enlargement of the cerebral sulci,

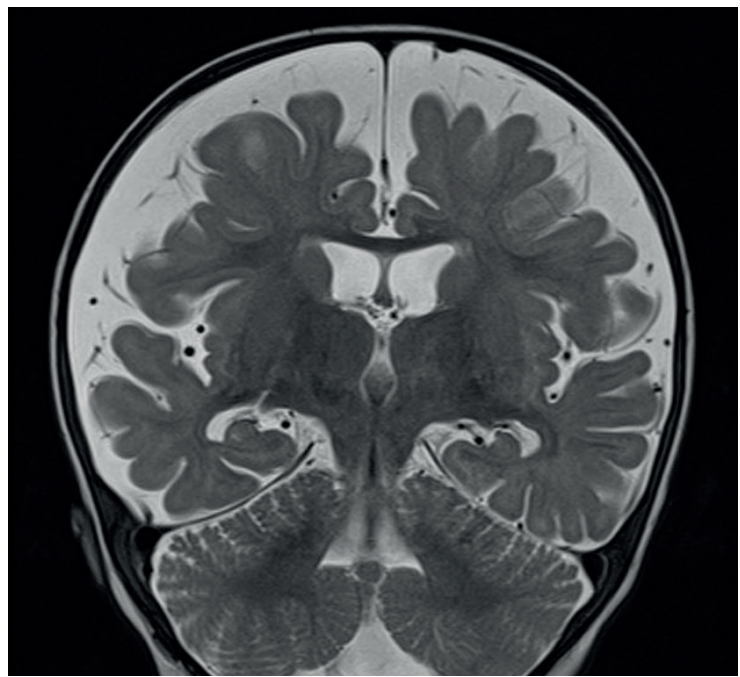

Fig. 1. Coronal T2-weighted image showing abnormal brain MRI findings including enlargement of frontotemporal subarachnoid spaces. and thinning of the cortex by age (Fig 1). Electroencephalogram (EEG) showed no seizure activity. Peroral cyanocobalamin 1,000 mcg/ day therapy due to vitamin B12 deficiency was initiated. Twelve hours after the initiation of the therapy, the patient developed a whole-body tremor and rhythmic myoclonic movements, titubation, and restlessness in the hands and feet. She was conscious of her environment despite being restless and uneasy. However, these symptoms disappeared during sleep. The following day, clonazepam $0.1 \mathrm{mg} / \mathrm{kg} /$ day was initiated due to the persistence of the tremor and then the complaints decreased gradually.

\section{Case 2}

A ten-month-old boy was referred to our clinic with an initial diagnosis of pancytopenia. This patient was also being breastfed. Although he was conscious of his environment, he was apathetic. He had been born at term with a birth weight of $3.2 \mathrm{~kg}$ after an uncomplicated pregnancy and delivery. He started to smile and control his head at 2 months of age. However, although he could hold his head, he could not sit without support. His head circumference was $43 \mathrm{~cm} \mathrm{(<3p),} \mathrm{body} \mathrm{weight} \mathrm{was} 9 \mathrm{~kg}(10 \mathrm{p})$, and body height was $70 \mathrm{~cm}$ (25p). He had several hyperpigmented lesions on the arm, back, sacral region and he had weak and sparse hair (Fig 2 $a, b, c)$. Cranial nerve examination was normal. All deep tendon reflexes were present and brisk. CBC parameters included hemoglobin $7.6 \mathrm{~g} / \mathrm{dL}$, hematocrit 26.2\%, MCV 82.1 fL, thrombocyte $132 \times 10^{3} / \mu \mathrm{L}$, ferritin $200 \mu \mathrm{g} / \mathrm{L}$, folic acid 8.56 $\mu \mathrm{g} / \mathrm{L}$, serum homocysteine $91 \mu \mathrm{mol} / \mathrm{L}$, and serum methylmalonic acid $3.85 \mu \mathrm{mol} / \mathrm{L}$. Both his and his mother's serum B12 vitamin levels were low (38 and $115 \mathrm{ng} / \mathrm{L}$, respectively). Peripheral blood analysis indicated hypersegmented neutrophils, anisocytosis, and poikilocytosis. A peroral cyanocobalamin $1000 \mathrm{mcg} /$ day supplementation therapy was initiated. Two days later, the tremor and rhythmic myoclonic movements in his feet were initially considered as seizures and antiepileptic treatment was started. Cranial magnetic resonance imaging 

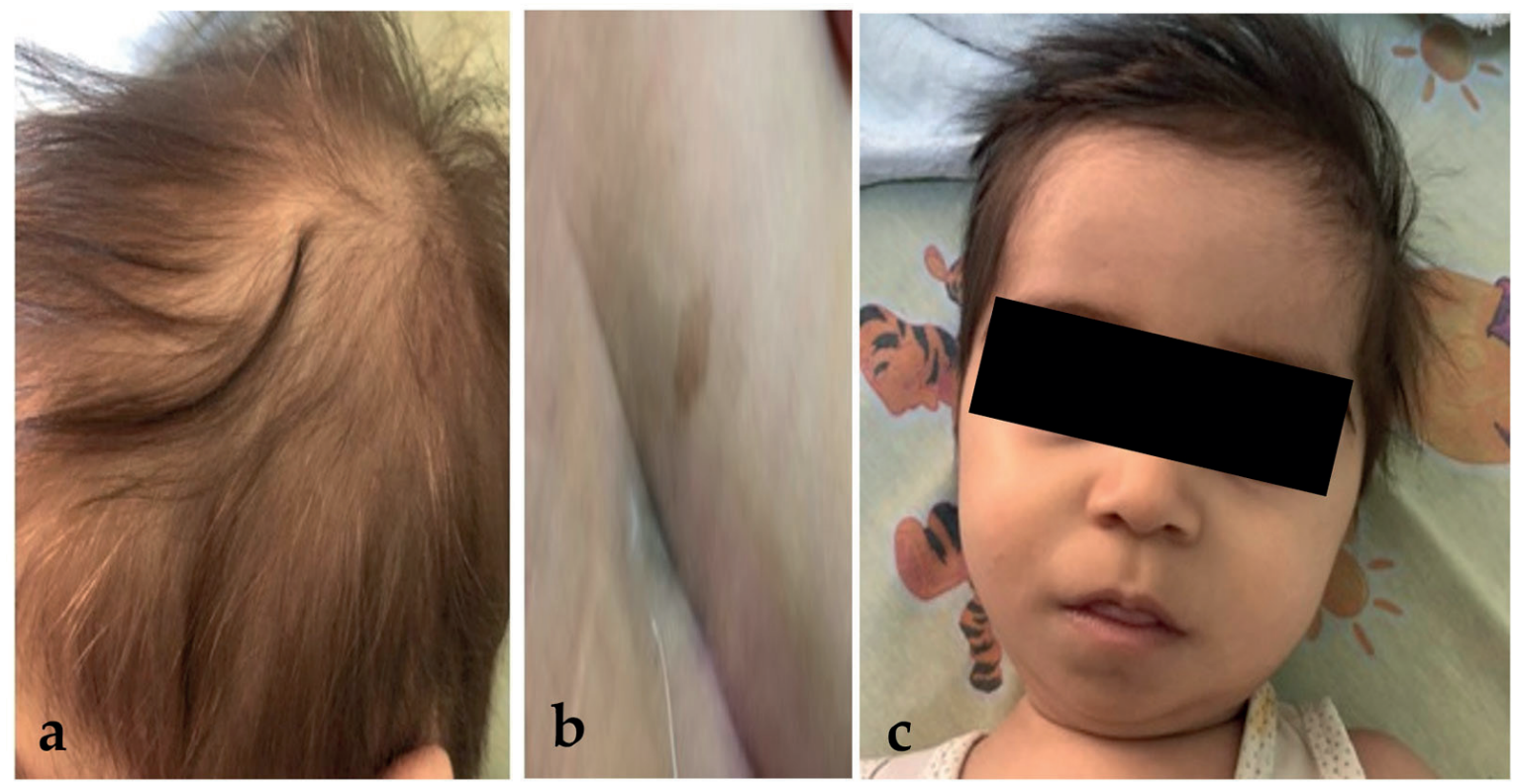

Fig. 2. Clinical image showing hypopigmented sparse hair, knuckle pigmentation, and apathy.

(MRI) visualized cerebral atrophy while EEG was normal. On the third day, a clonazepam therapy was added to the ongoing oral cyanocobalamin $1000 \mathrm{mcg} /$ day replacement therapy. Two days later, the complaint of tremor decreased gradually and the myoclonus disappeared completely.

\section{Discussion}

Vitamin B12 is an essential vitamin mostly ingested via animal products. Vitamin B12 deficiency can be seen at any age throughout life and remains a serious health problem both in Turkey and other developing countries. A recent study conducted in Turkey reported that the rates of maternal and infantile vitamin B12 deficiency were $76.7 \%$ and $60.8 \%$ during the first month post-delivery, respectively. ${ }^{1}$ On the other hand, although vitamin B12 deficiency has been reported extensively, the clinical spectrum of abnormal movements due to infantile B12 deficiency has been shown to be remarkably wide. ${ }^{9,10}$ ITS is characterized by paleness, growth retardation, tremor, hyperpigmentation, movement disorders, and sparse brown hair. ${ }^{11}$ Common neurological complications associated with vitamin B12 deficiency include hypotonia, optic atrophy, myelopathy, and lethargy. Tremor and myoclonus can be associated with vitamin B12 deficiency and may recur suddenly and worryingly after the initiation of vitamin B12 supplementation therapy. ${ }^{4,11-16}$ Nevertheless, the primary cause of abnormal movement that occurs after treatment is not fully known.

Chalouhi et al. ${ }^{16}$ suggested that overstimulation of the cobalamin-folate interaction leads to a metabolic disequilibrium. Emery et al. ${ }^{17}$ proposed that persistent hyperglycemia results in movement disorders during vitamin B12 treatment, and de Souza et al. ${ }^{18}$ reported that there are multiple factors associated with the pathophysiology of the hypersensitization effect of vitamin B12 deficiency, primarily including endothelial dysfunction induced by excitatory amino acids and increased methyltetrahydrofolate and homocysteine levels. ${ }^{16-18}$ Elevated homocysteine shows a similar effect at the N-methyl-Daspartate type of glutamate receptor and also activates the basal ganglia. ${ }^{19,20}$ Following vitamin B12 therapy, infants may present with tremor, myoclonus, and even seizures in addition to neurological symptoms. ${ }^{5}$ In such patients, EEG is often normal and is a significant source of information 
in the differential diagnosis of seizure-like symptoms., ${ }^{2,5}$ Various neuroradiological findings may be observed in cranial imaging in infants with vitamin B12 deficiency, namely including cortical atrophy, thinning of the corpus callosum, structural abnormalities, and retardation in myelination. Taşkesen et al. ${ }^{21}$ showed that thinning of the corpus callosum was detected in $6(40 \%)$, cortical atrophy in 5 $(33.3 \%)$, ventricular dilatation in $3(20 \%)$, large sylvian fissures in 5 (33.3\%), hydrocephalus in $3(20 \%)$, asymmetric large lateral ventricle in $2(13.3 \%)$, and retardation and myelination in $2(13.3 \%)$ patients while 4 infants had normal MRI findings. On the other hand, cranial imaging findings are highly useful in the differential diagnosis of patients that develop ITS following the initiation of vitamin B12 replacement therapy, particularly due to the risk of thromboembolism. ${ }^{22}$ In such patients, myelination problems and basal ganglia lesions are commonly seen and cerebral atrophy is the most common and reversible problem..${ }^{15}$ In our report, both patients presented with hypotonia, neuromotor and growth retardation, cerebral atrophy, and microcephaly. Moreover, abnormal movements such as myoclonic and tremors were also observed in both cases following peroral vitamin B12 replacement therapy. These findings were initially confused with seizure-like activity although EEG examinations were normal in both patients. Moreover, both patients were detected with bilateral cerebral frontotemporal atrophy and enlarged subarachnoid spaces on cranial MRI.

Nutritional vitamin B12 deficiency is effectively treated with peroral cyanocobalamin $1000 \mathrm{mcg} /$ $\mathrm{mL}$ (Dodex ${ }^{\circledR}$ ). ${ }^{23-25}$ Bahadir et al. ${ }^{25}$ noted that the vitamin B12 levels did not decrease in children aged less than two years during the long term after treatment. On the other hand, Sezer et al. ${ }^{23,24}$ found that the intramuscular and oral administration of cyanocobalamin had a similar effect, and this finding has been reported in several book chapters as well. In our clinic, vitamin B12 replacement therapy is routinely administered peroral due to the effectiveness and practicality of this approach.
Unless treated, infantile vitamin B12 deficiency may lead to irreversible problems. Almost $30 \%$ of cases of ITS may be accompanied by microcephaly and $75 \%$ of them may present with changes of pigmentation and sparse hair. ${ }^{11}$ In addition to these symptoms, abnormal movement may also be seen following vitamin B12 replacement therapy, as seen in both of our patients. Although a consensus on the role of Vitamin B12 deficiency in children with ITS is likely to be developed, it is still debatable. Moreover, it remains unclear as to why these abnormal movements often manifest as tremor. In such cases, myoclonus may also occur as a result of increased receptor synthesis. To our knowledge, all the ITS cases reported in the literature occurred after intramuscular administration of drugs while only one case in the series of Tunçer et al. ${ }^{12}$ occurred following peroral treatment.

In 2011, the Turkish Society of Hematology reported a guideline for B12 replacement therapy regardless of age and recommended an initial dose of 250-1000 $\mu \mathrm{g} /$ day B12 for the first week. The dose range is remarkably wide and the initial usage doses proposed in children vary across the studies in the literature. Sezer et al. ${ }^{24}$ demonstrated the effectiveness of the oral dose in their study, in which the treatment of 10 out of 142 children started at a dose of $100 \mathrm{mcg} /$ day and no side effect was observed. Yilmaz et al. ${ }^{26}$ indicated that they converted their treatments to hydroxocobalamin as a result of the oral cyanocobalamin treatment which was initiated at a dose of 250 mcg with 24 infants aged 2-18 months. Although the reason for the impairment in the initial treatment of cases may be caused by the use of high-dose cyanocobalamin, Bahadir et al..$^{25}$ started a treatment of $1000 \mathrm{mcg}$ cyanocobalamin in patients with a B12 level of $<50 \mathrm{pg} / \mathrm{ml}$ and observed no side effects in any patient. Although it is recommended that low-dose treatment initiation is a controlled condition to prevent the possible side effects such as movement disorders and other possible side effects that may occur after B12 deficiency treatment, there is a need for studies to show 
the likelihood of dose-related movement disorders in children. On the other hand, there is not a definite recommended dose for oral B12 replacement therapy particularly for infants.

In both of our patients, abnormal movements occurred following peroral vitamin B12 replacement therapy. Meaningfully, abnormal movements such as tremor and myoclonus may be observed during the B12 vitamin treatment or particularly due to vitamin B12 deficiency. We consider that more care should be taken for various movement disorders that may occur after the replacement therapy initiated in infants and their mothers with vitamin B12 deficiency. Knowledge of treatment options in such patients is of paramount importance since these cases are commonly encountered in clinical practice. Clonazepam $0.1 \mathrm{mg} / \mathrm{kg} /$ day is the mainstay treatment of ITS while propranolol $2 \mathrm{mg} / \mathrm{kg} /$ day, piracetam $4.8 \mathrm{~g} /$ day, and carbamazepine $10 \mathrm{mg} / \mathrm{kg}$ have been shown to be effective in ITS patients. ${ }^{8,27}$

Early diagnosis and treatment of vitamin B12 deficiency accompanied by growth retardation and macrocytic anemia are highly important for the prevention of infantile tremor. It should be noted that the infants of mothers with nutritional vitamin B12 deficiency may develop sudden abnormal movements following peroral vitamin B12 therapy and that the differential diagnosis of these disorders is highly important for the prevention of long-term neurological sequela by treatment. Moreover, extensive knowledge of the medications to be used in such patients is of paramount importance for the prevention of unnecessary drug use. Assessment and treatment of maternal vitamin B12 levels, especially in the last three months of pregnancy or after childbirth, is of vital importance for preventing myelination in the infants.

All the authors declare that they have no conflict of interests in this study. The study was not funded by any institution or establishment. A written consent was obtained from the parents of both patients.

\section{Author contribution}

Beril Dilber, and Gökçe Pınar Reis contributed to the medical care and diagnose for this study.

\section{Conflict of interest}

All the authors declare that they have no conflict of interests in this study.

\section{REFERENCES}

1. Çoban S, Yılmaz Keskin E, İğde M. Association between maternal and infantile markers of Cobalamin status during the first month postdelivery. Indian J Pediatr 2018; 85: 517-522.

2. Snow CF. Laboratory diagnosis of vitamin B12 and folate deficiency: a guide for the primary care physician. Arch Intern Med 1999; 28: 1289-1298.

3. MasriA. Eye myoclonus: A rare manifestation of vitamin B12 deficiency. J Pediatr Neurol 2006; 4: 4951.

4. Graham, SM, Arvela OM, Wise GA. Long-term neurologic consequences of nutritional vitamin B12 deficiency in infants. J Pediatr 1992; 121(5 Pt 1): 710714 .

5. Grattan-Smith PJ, Wilcken B, Procopis PG, Wise A. The neurological syndrome of infantile cobalamin deficiency: developmental regression and involuntary movements. Mov Disord 1997; 12: 39-46.

6. Stollhoff K, Schulte FJ. Vitamin B12 and brain development. Eur J Pediatr 1987; 146: 201-205.

7. Ozer EA, Turker M, Bakiler AR, Ozturk YC. etInvoluntary movements in infantile cobalamin deficiency appearing after treatment. Pediatr Neurol 2001; 25: 81-83

8. Ozdemir O, Baytan B, Gunes AM, Okan M. Involuntary movements during vitamin B12 treatment. J Child Neurol 2010; 25: 227-230.

9. Yaramis A. A variety of abnormal movements in 13 cases with nutritional cobalamin deficiency in infants. Med Hypotheses 2020; 142: 109796.

10. Taşkesen M, Yaramış A, Katar S, Gözü Pirinççioğlu A, Söker M. Neurological presentations of nutritional vitamin B12 deficiency in 42 breastfed infants in Southeast Turkey. Turk J Med Sci 2011; 41 1091-1096.

11. Gowda VK, Kolli V, Benakappa A, Srinivasan VM, Shivappa SK, Benakappa N. Case series of infantile tremor syndrome in tertiary care paediatric centre from Southern India. J Trop Pediatr 2018; 64: 284288. 
12. Tunçer GO, Köker A, Köker SA, et al. Infantile tremor syndrome after peroral and intramuscular vitamin B12 therapy: two cases. Klin Padiatr 2019; 231: 274-277.

13. Patiroglu T, Unal E, Yildirim S. Infantile tremor syndrome associated with cobalamin therapy: a case report. Clin Neurol Neurosurg 2013; 115: 1903-1905.

14. Avci Z, Turul T, Aysun S, Unal I. Involuntary movements and magnetic resonance imaging findings in infantile cobalamine (vitamin B12) deficiency. Pediatrics 2003; 112(3 Pt 1): 684-686.

15. Garewal G, Narang A, Das KC. Infantile tremor syndrome: a vitamin B12 deficiency syndrome in infants. J Trop Pediatr 1988; 34: 174-178.

16. Chalouhi $C$, Faesch $S$, Anthoine-Milhomme MC, Fulla Y, Dulac O, Chéron G. Neurological consequences of vitamin B12 deficiency and its treatment. Pediatr Emerg Care 2008; 24: 538-541.

17. Emery ES, Homans AC, Colletti RB. Vitamin B12 deficiency: a cause of abnormal movements in infants. Pediatrics 1997; 99: 255-256.

18. de Souza A, Moloi MW. Involuntary movements due to vitamin B 2 deficiency. Neurol Res 2014; 36: 1121-1128.

19. Berardelli A, Thompson PD, Zaccagnini M, et al. Two sisters with generalized dystonia associated with homocystinuria. Mov Disord 1991; 6: 163-165.

20. Kempster PA, Brenton DP, Gale AN, Stern GM. Dystonia in homocystinuria. J Neurol Neurosurg Psychiatry 1988; 51: 859-862.
21. Taşkesen M, Yaramış A, Gözü Pirinççioğlu A, Ekici F. Cranial magnetic resonance imaging findings of nutritional vitamin B12 deficiency in 15 hypotonic infants. Eur J Paediatr Neurol 2012; 16: 266-270.

22. Unal E, Mutlu FT, Karakukcu M. The importance of MTHFR polymorphisms in pediatric cerebral stroke. Child Nerv Syst 2012; 28: 13.

23. Sezer RG, Bozaykut A, Ayhan Akoğlu H, Özdemir GNç. The efficacy of oral vitamin B12 replacement for nutritional vitamin B12 deficiency. J Pediatr Hematol Oncol 2018; 40: e69-e72.

24. Sezer RG, Ayhan Akoğlu H, Bozaykut A, Özdemir GN. Comparison of the efficacy of parenteral and oral treatment for nutritional vitamin B12 deficiency in children. Hematology 2018; 23: 653-657.

25. Bahadir A, Reis PG, Erduran E. Oral vitamin B12 treatment is effective for children with nutritional vitamin B12 deficiency. J Paediatr Child Health 2014; 50: 721-725.

26. Yilmaz S, Serdaroglu G, Tekgul H, Gokben S. Different neurologic aspects of nutritional B12 deficiency in infancy. J Child Neurol 2016; 31: 565568.

27. Murali MV, Sharma PP, Koul PB, Gupta P. Carbamazepine therapy for infantile tremor syndrome. Indian Pediatr 1993; 30: 72-74. 